\title{
Phase Steps and Amplitude Fading of VLF Signals at Dawn and Dusk
}

\author{
D. Walker
}

\author{
Ministry of Ãviation, Royal Āircraft Establishment, S. Farnborough, Hampshire, England
}

(Received March 12, 1965; revised June 28, 1965)

\begin{abstract}
An experiment is described which tests the consequences of an explanation by Crombie of phase steps and amplitude fading at dawn and dusk. It is concluded that the explanation is valid at 18.0 $\mathrm{kc} / \mathrm{s}$ for the types of path studied.
\end{abstract}

\section{Introduction}

The diurnal phase variation of VLF signals over long paths is generally in the form of a trapezium [Pierce, 1955] where all night conditions over the path correspond to one steady-phase level, and all day conditions over the path correspond to the other steadyphase level. The idealized transition from one phase level to the other during dawn and dusk completes the straight sides of the trapezium, and this paper discusses deviations from this in the form of phase steps during the transition period. The phase steps are accompanied by signal fading, and this had in fact been noticed before transmitter frequencies were stable enough to detect the diurnal phase variations [Anderson, 1928]. Attempts were made to explain the amplitude fading by Yokoyama and Tanimura [1933] and the phase steps by Rieker [1963]. Both explanations were based on single-ray geometrical optics, but it has been pointed out by Budden [1961] and Wait [1962a] that many rays are needed to explain VLF propagation over very long paths. Crombie [1964] put forward an explanation based on the use of modes in the earthionosphere waveguide in which two modes are present in the nighttime part of the path and only one mode in daylight. He showed that this explanation fitted most of the observed facts and that it had consequences which were capable of verification by further experimental evidence.

An experiment is described in this paper which produced results in support of Crombie's explanation. The experiment involved the use of a mobile monitor station on board ship, and a permanent ground monitor station, both of which recorded the phase and amplitude of NBA 18.0-kc/s signals from Balboa, Canal Zone.

The consequences of Crombie's explanation are studied in more detail, and it is shown that the difference in phase velocity and the difference in attenuation rate for the two lowest order modes at night can be deduced from experimental data.

Information can also be obtained on the transmitter excitation factor and the mode conversion at the ionosphere discontinuity.
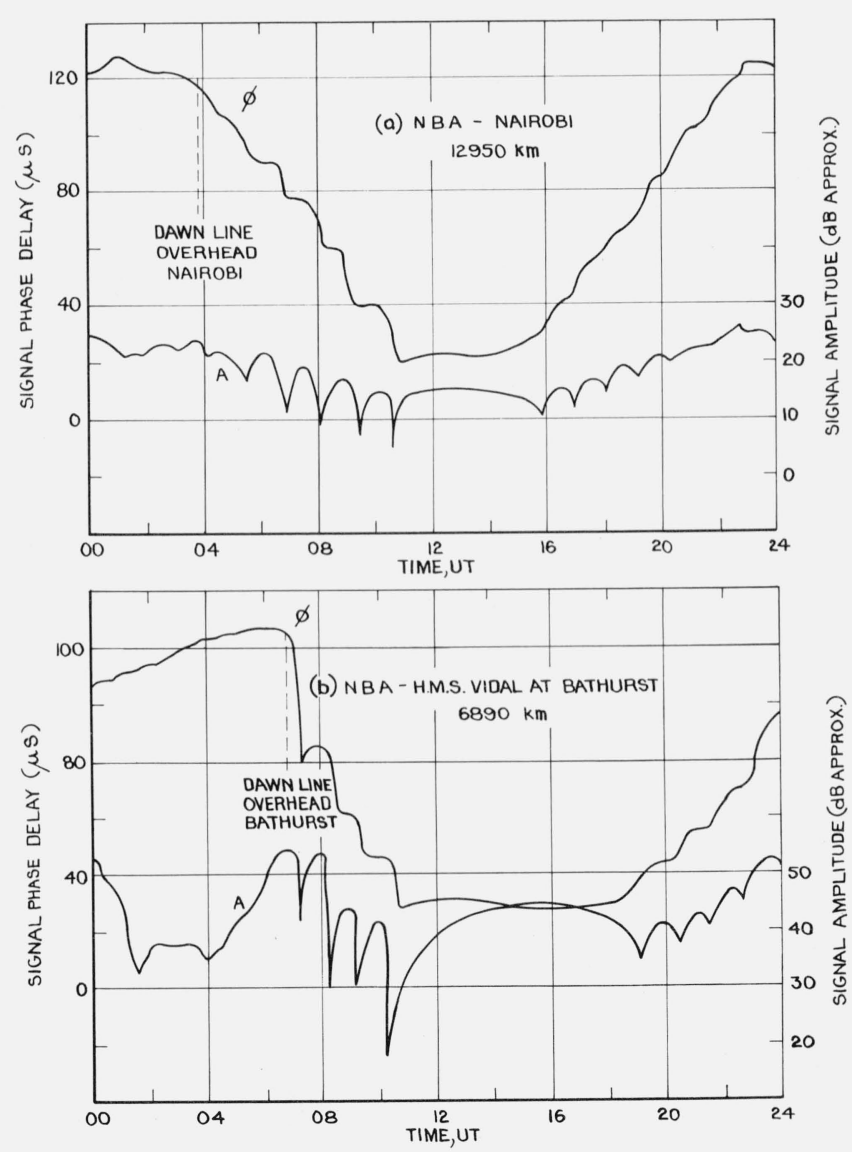

FigURE 1. NBA 18.0-kc/s phase ( $\phi)$ and amplitude (A) records, 6 December 1963.

\section{Explanation of Phase Steps Put Forward by Crombie}

Phase steps and signal fading similar to that illustrated in figure 1 were studied by Crombie [1964] on the transmissions of NPM $(19.8 \mathrm{kc} / \mathrm{s})$, Hawaii, to 
Boulder, Colo., and NBA (18.0 kc/s), Canal Zone, to Frankfurt, Germany. He noted that:

(1) The rapid change of phase took place at the same time as the amplitude minimum;

(2) the rapid phase change was in the direction of decreasing phase delay during sunrise, and of increasing phase delay during sunset (i.e., in the same direction as the general phase change at these times);

(3) during sunrise, the deepest minimum occurred latest in time, i.e., with the dawn line closest to the transmitter, but during sunset the evidence from the two paths was not so definite;

(4) NPM signals received at Boulder and at Washington (almost on a great-circle line from Hawaii) seemed to show that the minima during sunrise occurred at the same time at the two places, to within 5 min or less (only a few days' observations were available); and

(5) the distance moved by the dawn-dusk line along the transmitter-receiver paths between times of signal minima was the same for sunrise and sunset.

Crombie put forward a model similar to that illustrated in figure 2 to explain the observations. $\mathrm{He}$ assumed that at sunrise, for a west-to-east transmission, two waveguide modes are present in the dark part of the path between the transmitter and the dawn discontinuity, but that at the discontinuity the second mode is converted to first mode, so that no second mode exists in the daylight part of the path. He showed that the consequences of these assumptions are that when destructive interference takes place to give a voltage minimum, the rapid phase change is in the observed direction, and that all points to the east of the dawn discontinuity should experience the signal minimum simultaneously, which agrees with the few days' observations quoted above.

In order to explain the sunset phenomena, Crombie assumed that only the first waveguide mode exists between the transmitter and the discontinuity, but that some second mode is generated at the discontinuity, so that between this point and the receiver, in the dark part of the path, two modes are present, and an interference pattern is formed which moves westward with the sunset line. Crombie showed that with this model, the rapid phase change associated with the voltage minimum is again in the observed direction.

Crombie's model therefore fitted observations (1), (2), (4), and (5) (the latter, since in each case it is

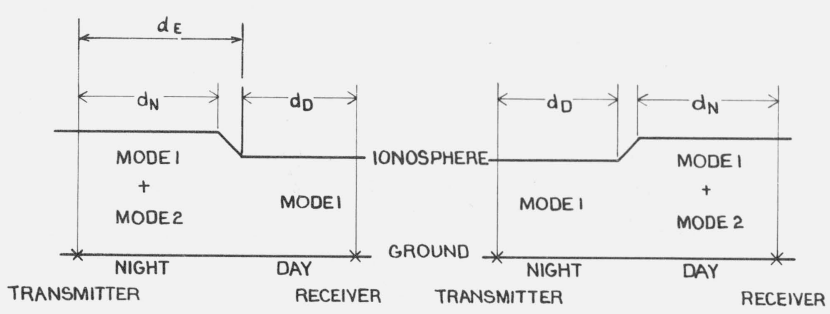

a) SUNRISE

b) SUNSET

FIGURE 2. Ionosphere model for sunrise and sunset. the nighttime height of ionosphere which determines the two mode velocities, and hence the distance moved by the dawn-dusk line between minima). However, the experimental evidence in support of the simultaneity of the minimum signal at sunrise was slight, and none was available to show that at sunset the interference pattern moves with the sunset line. Thus the explanation, while fitting the general facts, had not been verified by any substantial amount of experimental evidence.

The principal object of this paper is to describe an experiment in which the main consequences of Crombie's explanation have been verified. The experiment is described in section 3 , and the analysis of the results is discussed in section 4 .

The consequences of the explanation by Crombie are discussed more fully by taking into account the transmitter excitation factor, the mode conversion coefficient at the dawn-dusk discontinuity, and the attenuation rates of the first two waveguide modes. None of these factors were considered in detail by Crombie. The relationships derived are applied to the experimental data in section 4 to give the difference in phase velocity and the difference in attenuation rate for the first two modes at night. By assuming a theoretical value for the excitation factors of mode 1 and mode 2 at night, an estimate is made of the ratio of the two lowest order mode conversion coefficients at the dawn discontinuity.

\section{Experimental Data}

The two main consequences of Crombie's explanation of the dawn-dusk phase steps are that, for a W-to-E path,

(1) At dawn, all points to the east of the discontinuity should experience the signal minima simultaneously;

(2) at dusk, the interference pattern should be on the dark side of the discontinuity and should move with the discontinuity so that the time of occurrence of minimum signal should depend on the location of the receiver.

The verification of both of these results could be done ideally by placing a large number of receivers at intervals along a great-circle path from a suitable transmitter, and simultaneoulsy recording the phase and amplitude of the signal at all the points. This experiment was not feasible, but a permanent recording station at a great distance from a transmitter, together with a mobile recording station moving to a different position each day along the great-circle path between the transmitter and the permanent monitor station, is a good approximation to the ideal, and this experiment has been done.

A permanent monitor station is in operation at the University of East Africa, Nairobi, Kenya, and the phase of received VLF signals is compared with the phase derived from a rubidium-vapor frequency standard. The phase difference is recorded together with signal amplitude, and the phenomenon of phase steps and amplitude fading can be seen clearly on the NBA $18.0-\mathrm{kc} / \mathrm{s}$ records, an example of which is given in figure la. The path length is $12,950 \mathrm{~km}$. The 


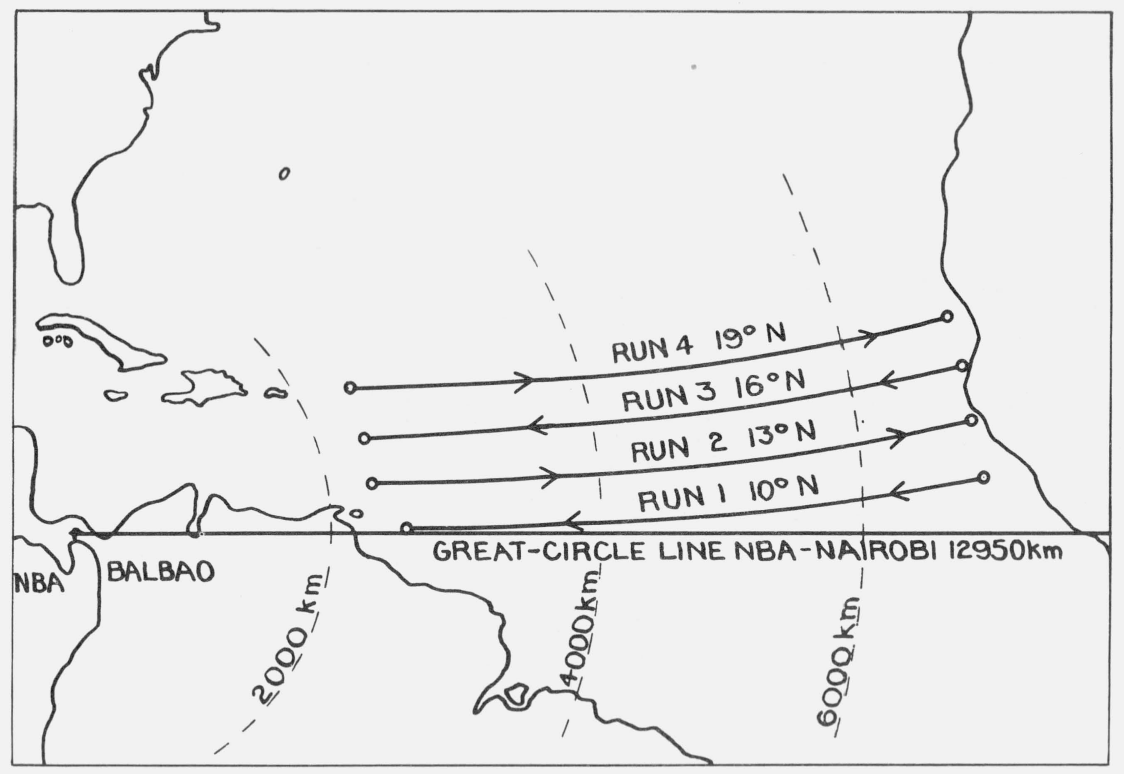

Figure 3. Path followed by H.M.S. Vidal during Exercise Navado.

dawn steps are always very noticeable, the number varying between four and seven but those at dusk are much less pronounced and are sometimes masked by locally generated noise. Recording paper speeds of $15 \mathrm{~mm} / \mathrm{hr}$ enable the timing of events to be estimated to better than 4 min. Records were available from 11 November to 31 December 1963, which covers most of the time that the mobile monitor station on H.M.S. Vidal was in operation.

H.M.S. Vidal is a Naval survey ship engaged on oceanographic survey work, and during Exercise Navado a temporary VLF monitor station was established on board. The station was equipped in the same manner as the permanent station at Nairobi, with the exception that a vertical whip aerial was used instead of the loop aerial used at the ground recording station. Amplitude minima and phase steps can be seen in figure $1 \mathrm{~b}$ which shows phase and amplitude readings of NBA $18.0 \mathrm{kc} / \mathrm{s}$ taken on H.M.S. Vidal with the ship stationary, on the same date as the Nairobi record in figure la. While the ship was in motion, the phase steps were superimposed on the phase change caused by the movement of the ship relative to the transmitter, and were sometimes difficult to interpret, but the amplitude minima were still clear. The speed of the recording paper was increased to $60 \mathrm{~mm} / \mathrm{hr}$ in order to ease the reading of phase records made while the ship was moving, and in this case the time of any event can be read to the nearest minute. Exercise Navado consisted of four crossings of the Atlantic on fixed lines of latitude of $10^{\circ}, 13^{\circ}, 16^{\circ}$, and $19^{\circ} \mathrm{N}$. The ship's speed was maintained as nearly constant as possible at 12 knots for 2 days, followed by $12 \mathrm{hr}$ "on station" with engines stopped. Astronomical fixes were taken twice a day so that the ship's position was known to an accuracy of about 1 mile at any time. The first three crossings, made between 30 October and 21 December 1963 , produced good quality VLF data, and this has been used in the subsequent analysis. The path of the ship in relation to the NBA transmitter and the Nairobi receiver is illustrated in figure 3 . The distance of the ship from the transmitter varied between $2000 \mathrm{~km}$ and $7000 \mathrm{~km}$.

\section{Analysis of Results}

\subsection{General}

The experimental measurements available were the times at which the signal minima (or maximum rate of change of phase) occurred at the different receiver positions, and the relative signal amplitude during the dawn and dusk period. The measurements of time are not significant in themselves, since they are dependent on the time of year, and on the location of the transmitter-receiver path. A more convenient parameter to use is the position of the dawn-dusk discontinuity at the time of the signal minimum, and its distance from the transmitter or receiver. The

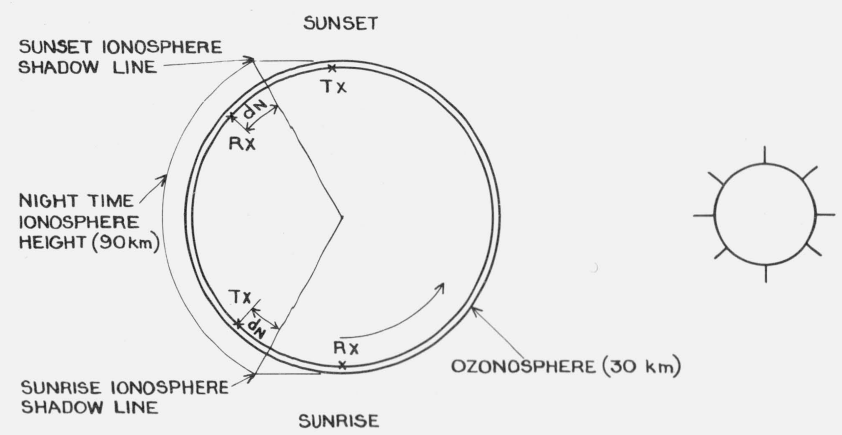

FIGURE 4. Ionosphere shadow line. 
position of the discontinuity in the height of the $D$ region of the ionsophere is not known exactly, but for convenience it has been defined here as the shadow cast by a $30-\mathrm{km}$ ozonosphere on a $90-\mathrm{km}$ ionosphere (see fig. 4), giving an angular position measured from the center of the earth. The same angular position would be obtained by taking the shadow line cast by the solid earth on a $60-\mathrm{km}$ ionosphere, so in this sense the definition is arbitrary, but it does define a line on the surface of the earth which bears a fixed relation to the true discontinuity. An examination of the NBA $18.0-\mathrm{kc} / \mathrm{s}$ phase records in figure 1 shows that the dawn variation starts when this shadow line is almost overhead at the receiver, and hence the definition used is not unreasonable for dawn conditions. At dusk, the phase change continues for approximately $2 \mathrm{hr}$ after the defined shadow line has passed overhead at the transmitter, showing that the discontinuity at dusk is not so sharp and not so close to the defined line as at dawn. Distances have been calculated from the position on the surface of the earth directly under the shadow line, as defined above, to the transmitter and the receiver, along the great-circle path between transmitter and receiver. A correction was made to the universal time at which the minima occurred, to allow for the equation of time [H.M. Nautical Almanac Office, 1961] which gives the difference between the true hour angle of the sun, and the hour angle of the ficticious mean sun, i.e.,

$$
H=12 \mathrm{hr}+\mathrm{UT}+E
$$

where $H$ is the Greenwich hour angle of the sun, UT is the universal time, and $E$ is the equation of time correction.

The limits of the magnitude of $E$ during a year are approximately $\pm 15 \mathrm{~min}$, which is equivalent to \pm 400 $\mathrm{km}$ in the position of the dawn-dusk discontinuity, and during the experiment the change in $E$ was approximately $17 \mathrm{~min}$, equivalent to $500 \mathrm{~km}$.

\subsection{Verification of Crombie's Explanation}

\section{a. Sunrise}

During the experiment, the mobile monitor station moved a distance of $5000 \mathrm{~km}$ in approximately 11 days on almost a great-circle path from the NBA transmitter, in each of three crossings of the Atlantic. The distance of the dawn ionosphere shadow line from the NBA 18.0-kc/s transmitter at times of minimum signal received by H.M.S. Vidal, as a function of distance between transmitter and receiver, is shown in figure 5 . It is seen that the minima occur when the dawn line is at discrete distances from the transmitter of approximately $500 \mathrm{~km}, 2500 \mathrm{~km}$, and $4400 \mathrm{~km}$, independent of the distance of the transmitter from the receiver. This result is an agreement with Crombie's explanation of the phenomenon. The number of minima decreases as the receiver approaches the transmitter, and when the receiver is near the distance from the transmitter at which the dawn line normally gives a minimum, an

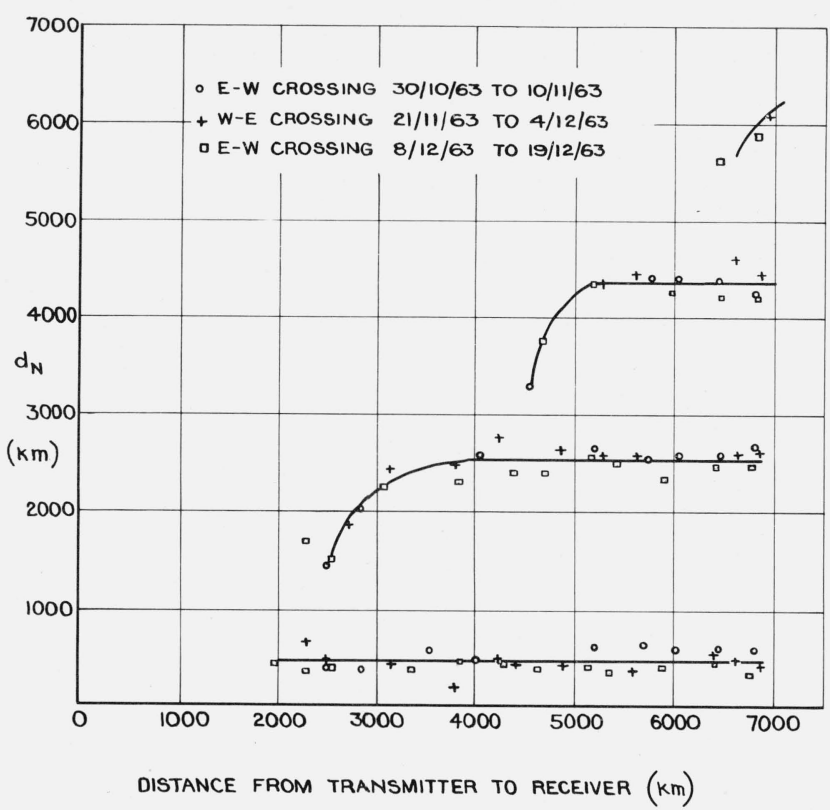

FIGURE 5. Distance $\mathrm{d}_{\mathrm{N}}$ of dawn line from NBA 18.0-kc/s transmitter at times of minimum signal for varying receiver positions.

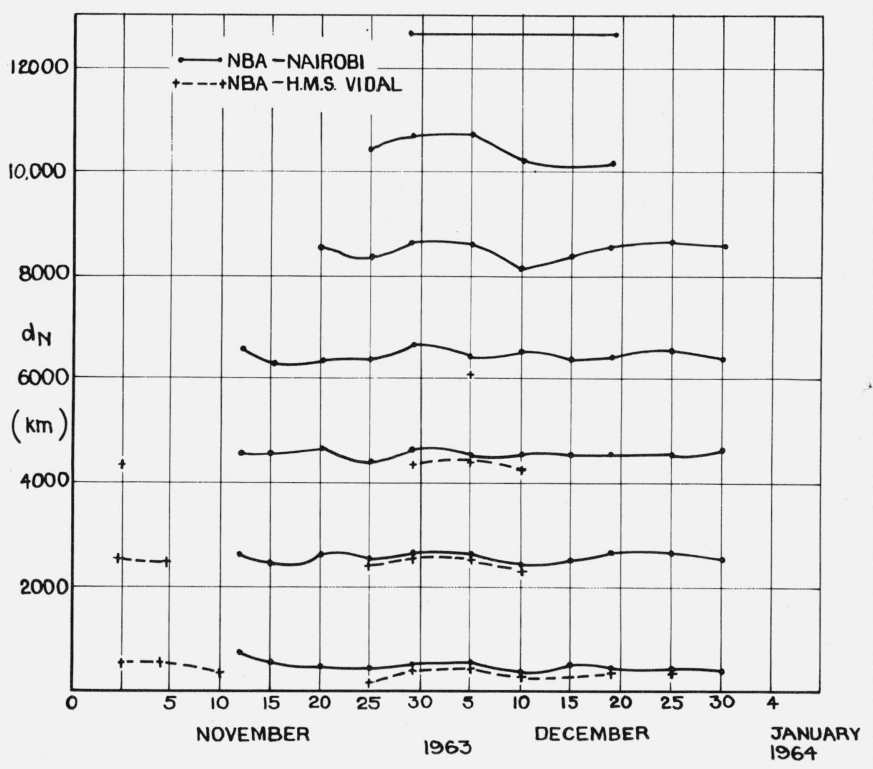

FiguRE 6. Distance $\mathrm{d}_{\mathrm{N}}$ of dawn line from NBA 18.0-kc/s transmitter at times of minimum signal at Nairobi and H.M.S. Vidal, November-December 1963.

anomalous condition occurs. In this case the minimum occurs at a later time than for receiver positions further from the transmitter, i.e., the dawn line is closer to the transmitter by approximately $1000 \mathrm{~km}$. A possible explanation is that, for receiver positions close to the dawn discontinuity, some second mode exists in the light part of the path, thus affecting the time of occurrence of the minimum signal, whereas for greater distances from the discontinuity the attenuation of the second mode is too great for it to have any appreciable effect. 
The fact that the results from three crossings of the Atlantic show no appreciable variation implies that the seasonal variation is small during this period of time, and this is also supported by the data from the fixed monitor station at Nairobi. This is illustrated in figure 6, which shows the distance of the dawn shadow line from the NBA transmitter at times of minimum signal received in Nairobi during NovemberDecember 1963. The mobile receiver results are also shown in figure 6 , and it is seen that many of the small day-to-day variations in the position of the dawn line for minimum signal are repeated at the fixed and mobile receiver positions, i.e., the variations are real and not inconsistencies in the data.

\section{b. Sunset}

For sunset, the consequences of Crombie's explanation are that the interference pattern is in the dark part of the path and moves along with the sunset line. Thus minima should occur with the sunset line at fixed distances from the receiver, independent of the transmitter-receiver displacement. Figure 7 shows the distance of the sunset line from the receiver at times of minimum NBA $18.0-\mathrm{kc} / \mathrm{s}$ signal received by H.M.S. Vidal during two crossings of the Atlantic. It is seen that the minima occur when the sunset line is at discrete distances from the receiver of approximately $100 \mathrm{~km}, 2000 \mathrm{~km}$, and $3800 \mathrm{~km}$. The scatter is greater than in the sunrise case, but the result is again in agreement with Crombie's explanation. The results from two crossings of the Atlantic separated by 14 days are in reasonable agreement, and the results from the fixed monitor station at Nairobi during

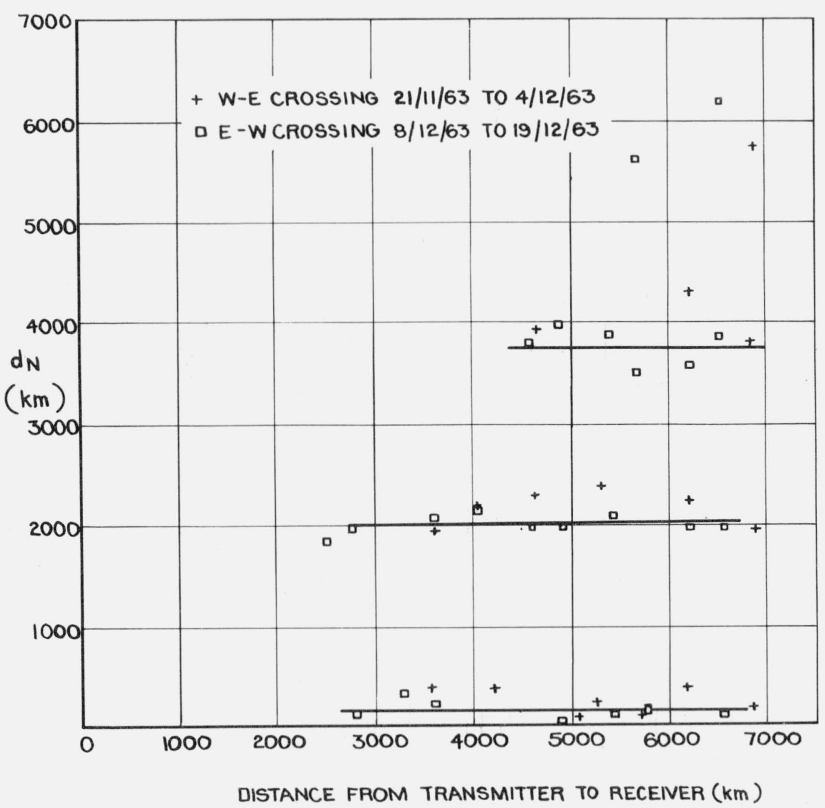

FiguRE 7. Distance $\mathrm{d}_{\mathrm{N}}$ of sunset line from receiver at times of minimum NBA 18.0-kc/s signal for varying receiver positions.

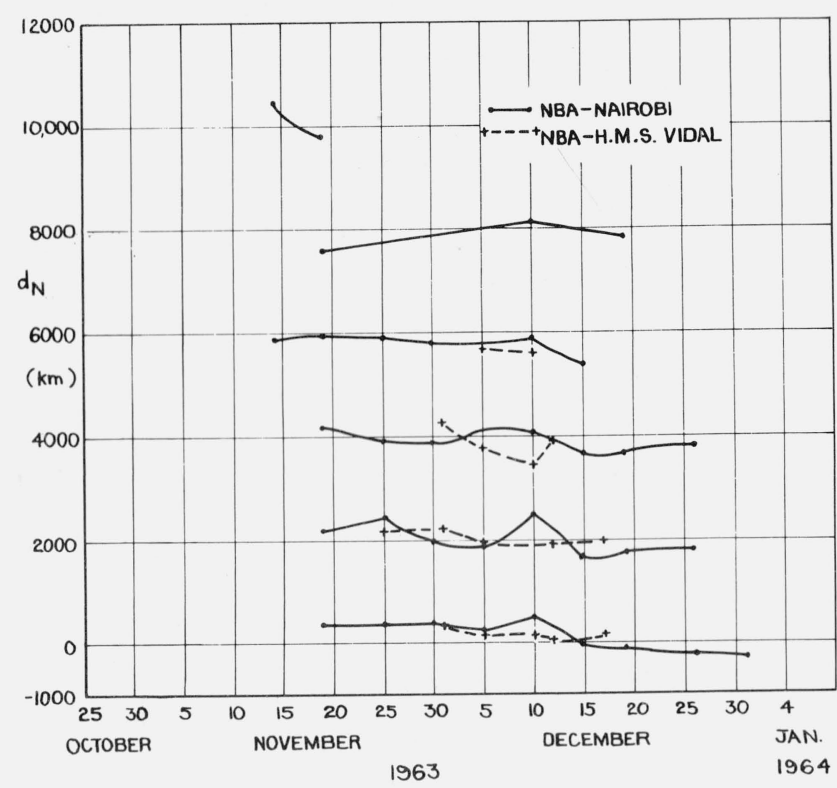

FigURE 8. Distance $\mathrm{d}_{\mathrm{N}}$ of sunset line from Nairobi and H.M.S. Vidal receivers at times of mimimum NBA 18.0-kc/s signal, November-December 1963.

November-December 1963 (see fig. 8) show that although there seems to be a slow seasonal variation, the change was small during the 11 days required for one crossing of the Atlantic.

\subsection{Phase Velocity}

The distance spacing, $D$, between the positions of the dawn-dusk line for minimum signal gives a measure of the phase velocity difference of the two interfering modes, and it can be shown that:

$$
\left(V_{N 2} / C-V_{N 1} / C\right) \doteqdot \lambda / D,
$$

where $V_{N 1}$ and $V_{N 2}$ are the phase velocities of mode 1 and mode 2 in darkness, and $\lambda$ is the free space wavelength at $18.0 \mathrm{kc} / \mathrm{s}$. For the dawn case, the mean value of $D$ was $2000 \mathrm{~km}$, giving $\left(V_{N 2} / C-V_{N 1} / C\right)=0.0083$. For the dusk case, the mean value of $D$ was $1900 \mathrm{~km}$, giving $\left(V_{N 2} / C-V_{N 1} / C\right)=0.0088$. Theoretical values derived by Wait [1962a] for $\left(V_{2} / C-V_{1} / C\right)$ at 18.0 $\mathrm{kc} / \mathrm{s}$, for a perfectly conducting spherical earth and for a sharply bounded ionosphere with a value of conductivity parameter $\omega_{r}=2 \times 10^{5}$, are shown in figure 9 as a function of ionosphere height. From this it can be seen that the equivalent ionosphere height during dawn interference is $87.5 \mathrm{~km}$ and the equivalent ionosphere height during dusk interference is 84.5 $\mathrm{km}$. These values would be changed slightly if allowance were made for the effect of the earth's magnetic field, and for a gradual ionosphere boundary, but the result would still be consistent with the normally accepted ionosphere reflection height for VLF waves at night. This result is again in accordance with Crombie's explanation, since it implies that 


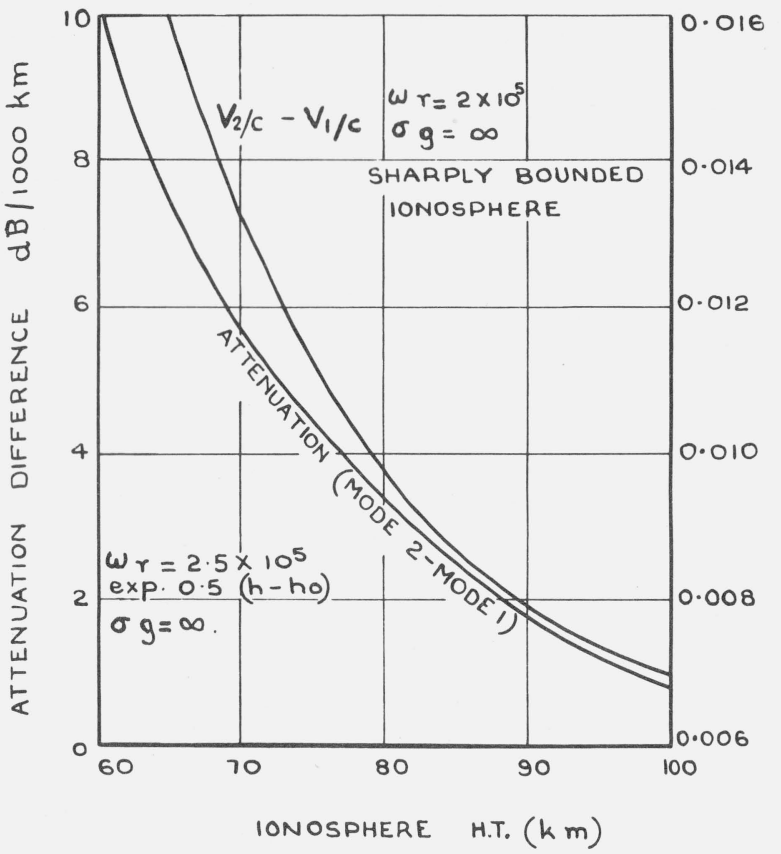

FIGURE 9. Theoretical values for difference in phase velocity [W ait, $1962 a$ ] and for difference in attenuation rate [Wait and Spies, 1964] of mode 1 and mode 2 at $18.0 \mathrm{kc} / \mathrm{s}$.

the interference is determined by the nighttime part of the path in each case. The slightly lower height at dusk may be due to the incomplete change to full nighttime conditions close to the dusk line.

\subsection{Position of Dawn-Dusk Line}

The distance of the dawn line from the transmitter at times of minimum signal gives information on the phase of excitation of the first two modes by the transmitter at night, and on the phase change introduced by the discontinuity.

Thus, referring to figure 2 , we assume that for $d$ $<d_{N}$, i.e., in the dark part of the path, we have a series of modes whose propagation is determined by the nighttime ionosphere reflection height $h_{N}$ and by the properties of the nighttime reflecting boundary. The field $E$ at a distance $d$ from a vertical electric dipole of strength $I d s$ is given as a sum of modes by [see Wait, 1962a]

$E=\frac{\eta i I d s}{\left(a \sin \frac{d}{a}\right)^{1 / 2} h_{N} \lambda^{1 / 2}} \sum_{n} \Lambda_{n} e^{-i k s_{N n} d} \quad 0<d<d_{N}$,

where

$\eta=(\mu / \epsilon)^{1 / 2} \doteqdot 120 \pi$ for free space,

$\lambda=$ free space wavelength,

$a=$ radius of earth, $n=$ mode number,

$\Lambda_{n}=$ transmitter excitation factor of mode $n$,

$$
k=2 \pi / \lambda \text {. }
$$

$S \doteqdot\left(\nu+\frac{1}{2}\right) / k a$

$\nu=$ pole in complex $\nu$ plane resulting from Watson transformation. Subscript $N n$ refers to $n$th mode at night.

We then assume that at the discontinuity some mode conversion takes place, so that for $d>d_{E}$, i.e., in daylight, we have a new series of modes, whose magnitude is determined by the mode conversion, and whose propagation is determined by the daytime ionosphere reflection height $h_{D}$ and by the properties of the daytime boundary, i.e..

$$
E=\frac{A}{\left(a \sin \frac{d}{a}\right)^{1 / 2} h_{D}} \sum_{m} \Gamma_{m} e^{-i k S_{D m}\left(d-d_{E}\right)} \quad d>d_{E},
$$

where $A=\frac{\eta i I d s}{\lambda^{1 / 2}}$,

$m=$ daytime mode number,

$d_{E}=$ distance from transmitter to end of discontinuity.

Subscript $D m$ refers to $m$ th mode by day,

$$
\Gamma_{m}=\sum_{n} T_{n m} \Lambda_{n} e^{-i k S_{N n} d_{N}}
$$

$d_{N}=$ distance from transmitter to start of discontinuity, and

$T_{n m}=$ the (complex) mode conversion coefficient from mode $n$ in darkness to mode $m$ in daylight.

If we now consider a position of the dawn discontinuity far enough from the transmitter for modes higher than the first two to be negligible, and a distance of the receiver far enough from the end of the discontinuity for modes higher than the first mode to be negligible, then

$$
\begin{array}{r}
E=\frac{A}{\left(a \sin \frac{d}{a}\right)^{1 / 2} h_{D}} \Gamma_{1} e^{-i k S_{D_{1}}\left(d-d_{E}\right)} \quad d>d_{E} \\
=\frac{A}{\left(a \sin \frac{d}{a}\right)^{1 / 2} h_{D}} e^{-i k S_{D_{1}} d_{D}\left[T_{11} \Lambda_{1} e^{-i k S_{N_{1}} d_{N}}\right.} \\
+T_{21} \Lambda_{2} e^{\left.-i k S_{N 2} d_{N}\right]}
\end{array}
$$

where $d_{D}=d-d_{E}=$ length of path in daylight.

If we now ignore the slowly varying quantities $(a \sin d / a)^{-1 / 2}$ and $e^{-i k S_{D 1} d_{D}}$, then voltage minima will 
occur when

$\arg \left(\Lambda_{1} T_{11} / \Lambda_{2} T_{21}\right)+2 \pi d_{N}\left(1 / \lambda_{N 1}-1 / \lambda_{N 2}\right)$

$$
=(2 p+1) \pi, p=0,1 \ldots .
$$

The distance $D$ moved by the dawn line between successive times of minimum amplitude is given by

$$
1 / D=1 / \lambda_{N 1}-1 / \lambda_{N 2} \doteqdot\left(V_{N 2} / C-V_{N 1} / C\right) / \lambda,
$$

where $\lambda$ and $V$ are wavelength and phase velocity. Putting $p=0$ in (1) gives

$$
\begin{aligned}
\arg \left(\Lambda_{1} T_{11} / \Lambda_{2} T_{21}\right) & =\pi-2 \pi d_{N}\left(1 / \lambda_{N 1}-1 / \lambda_{N 2}\right) \\
& =\pi-2 \pi d_{N} / D
\end{aligned}
$$

where $d_{N}$ is now the distance of the dawn line from the transmitter at the time of the last minimum in a dawn sequence.

The value of $D$ is relatively insensitive to the definition used to locate the discontinuity, but the value of $d_{N}$ depends directly on this definition. The distance $d_{N}$ is the distance from the transmitter to the start of the discontinuity (see fig. 2a), and this is the position which the ionosphere shadow line attempts to define. From figure $6, D$ and $d_{N}$ can be estimated, and we obtain a value of $\arg \left(\Lambda_{1} T_{11}\right) /\left(\Lambda_{2} T_{21}\right)=\pi / 2$. If we now assume a perfectly conducting ground, and a perfectly reflecting ionosphere, then the value of $\arg \left(\Lambda_{1} / \Lambda_{2}\right)$ is zero, and $\arg \left(T_{11} / T_{21}\right)=\pi / 2$. This is now the difference in phase for the first two modes, introduced between the start of the discontinuity as defined by the shadow line, and the end of the discontinuity, which it is unnecessary to define. In general, $\Lambda_{1}$ and $\Lambda_{2}$ will not be real, since the ionosphere model giving the best agreement with experimental data is not perfectly reflecting, and hence the value of $\arg \left(T_{11} / T_{21}\right)$ will be modified.

At dusk, we assume that only mode 1 is present between the transmitter and the start of the discontinuity in daylight, and that mode 1 and mode 2 are present beyond the discontinuity in darkness. By similar reasoning to that used for the dawn case it can then be shown that at a receiver beyond the discontinuity, the signal voltage $E$ is given by

$$
E=\frac{A}{(a \sin d / a)^{1 / 2}} \frac{1}{h_{N}} \Lambda_{1}^{\prime} e^{-i k S_{D 1} d_{D}}
$$

$$
\left[U_{11} e^{-i k S_{N 1} d_{N}}+U_{12} e^{-i k S_{N 2} d_{N}}\right]
$$

where $\Lambda_{1}^{\prime}$ is the excitation factor of mode 1 in daylight, $U_{n m}$ is the (complex) conversion coefficient from mode $n$ to mode $m$ at the dusk discontinuity, and distances $d_{D}$ and $d_{N}$ refer to lengths of paths in daytime and nighttime conditions, respectively.

If we again ignore the slowly varying quantities ( $a$ sin $d / a)^{-1 / 2}$ and $\exp \left(-i k S_{D 1} d_{D}\right)$, minimum signal voltage will occur when

$$
\begin{aligned}
\arg \left(U_{11} / U_{12}\right)+2 \pi d_{N}( & \left.1 / \lambda_{N 1}-1 / \lambda_{N 2}\right) \\
& =(2 p+1) \pi, p=0,1, \ldots .
\end{aligned}
$$

The distance $D$ moved by the dusk line between successive times of minimum amplitude is given by:

$$
1 / D=1 / \lambda_{N 1}-1 / \lambda_{N 2} \doteqdot\left(V_{N 2} / C-V_{N 1} / C\right) / \lambda,
$$

and putting $p=0$ in (2) we have

$$
\begin{aligned}
\arg \left(U_{11} / U_{12}\right) & =\pi-2 \pi d_{N}\left(1 / \lambda_{N 1}-1 / \lambda_{N 2}\right) \\
& =\pi-2 \pi d_{N} / D
\end{aligned}
$$

where $d_{N}$ is the distance of the dusk line from the receiver at the time of the first minimum in a dusk sequence. At dusk, the distance $d_{N}$ in figure $2 \mathrm{~b}$ is from the end of the discontinuity to the receiver, and once again it is this point that the shadow line attempts to define. From figure $8, D$ and $d_{N}$ can be estimated and we have

$$
\arg \left(U_{11} / U_{12}\right)=\frac{9}{10} \pi .
$$

This is the difference in phase for the first two modes introduced between the undefined beginning of the discontinuity and the end of the discontinuity as defined by the ionosphere shadow line.

\subsection{Depth of Minima}

The ratio of the maximum to minimum received signal gives a measure of the ratio of the amplitudes of the two interfering signals, and also of their relative attenuation rates with distance. Thus it follows from the assumptions made in section 4.4 that at dawn, the amplitude of signal when $d_{N}=d_{\mathrm{MAX}}$ is given by

$$
\begin{aligned}
& E_{\mathrm{MAX}}=\frac{A}{(a \sin d / a)^{1 / 2}} \frac{1}{h_{D}} e^{-i k S_{D 1} d_{D}} \\
& \times\left[\left|\Lambda_{1} T_{11}\right| e^{-\alpha_{N 1} d_{\mathrm{MAX}}}+\left|\Lambda_{2} T_{21}\right| e^{-\alpha_{N 2} d_{\mathrm{MAX}}}\right],
\end{aligned}
$$

and at $d_{N}=d_{\mathrm{MIN}}$ by

$$
\begin{aligned}
E_{\mathrm{MIN}}=\frac{A}{(a \sin d / a)^{1 / 2}} & \frac{1}{h_{D}} e^{-i k S_{D 1} d_{D}} \\
& \times\left[\left|\Lambda_{1} T_{11}\right| e^{-\alpha_{N 1} d_{\mathrm{MIN}}}-\left|\Lambda_{2} T_{21}\right| e^{-\alpha_{N 2} d_{\mathrm{MIN}}}\right] .
\end{aligned}
$$

Thus if the equivalent values of $E_{\mathrm{MAX}}$ and $E_{\mathrm{MIN}}$ are found at the same value of $d_{N}$, then

$$
\frac{E_{\mathrm{MAX}}+E_{\mathrm{MIN}}}{E_{\mathrm{MAX}}-E_{\mathrm{MIN}}}=\left|\frac{\Lambda_{1} T_{11}}{\Lambda_{2} T_{21}}\right| e^{d_{N}\left(\alpha_{N 2}-\alpha_{N 1}\right)} .
$$

(Attenuation factor $\alpha_{N n}$ is given by $\alpha_{N n}=-k \operatorname{Im} S_{N n}$.) 


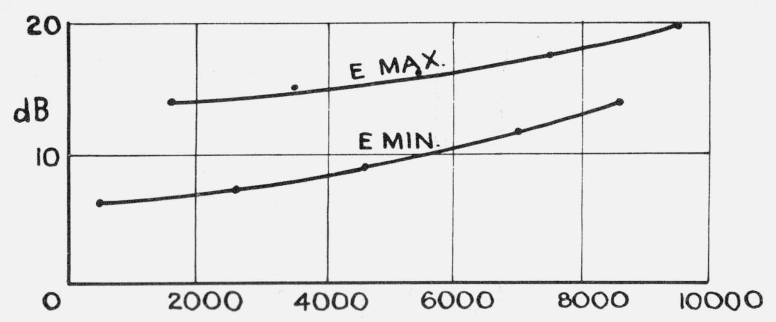

dN, DISTANCE OF DAWN LINE FROM TRANSMITTER $(\mathrm{km})$

a) MEAN MAXIMUM AND MINIMUM SIGNALS

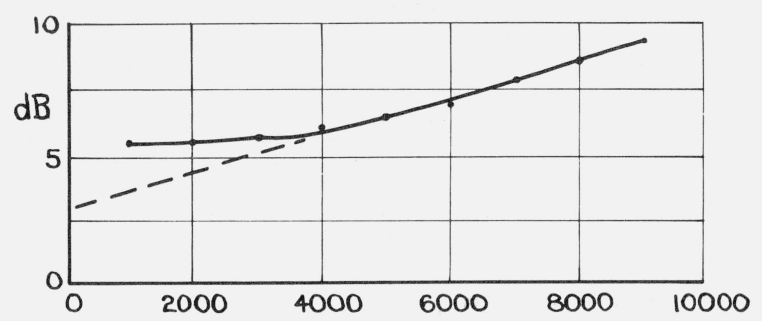

dN, DISTANCE OF DAWN LINE FROM TRANSMITTER $(\mathrm{km})$

b) $\frac{E M A X+E M I N}{E M A X-E M I N}$

FiguRE 10. NBA 18.0-kc/s dawn amplitudes at Nairobi, December 1963.

By plotting in figure 10a the mean values for the month of December 1963, of the dawn signal minima and signal maxima received at Nairobi, against $d_{N}$, the distance of the dawn line from the transmitter at the appropriate times, values of $E_{\mathrm{MAX}}$ and $E_{\mathrm{MIN}}$ for constant $d_{N}$ can be estimated. This enables values of $\left(E_{\mathrm{MAX}}+E_{\mathrm{MIN}}\right) /\left(E_{\mathrm{MAX}}-E_{\mathrm{MIN}}\right)$ to be calculated, and these are plotted in decibels against distance $d_{N}$ in figure $10 \mathrm{~b}$. From (3), the slope in figure $10 \mathrm{~b}$ gives $\left(\alpha_{N 2}-\alpha_{N 1}\right)$ in $\mathrm{dB} /$ unit distance, the intercept gives $\left|\Lambda_{1} T_{11} / \Lambda_{2} T_{21}\right|$ in $\mathrm{dB}$. There is a reduction in slope for $d_{N}<5000 \mathrm{~km}$ that may be due to the difficulty in estimating the correct minimum values in this range because of low signal strength. (Alternatively, the attenuation may be changed due to the presence of more than two modes in the dark path, when the dawn line is close to the transmitter.) If the slope beyond $5000 \mathrm{~km}$ is taken to be the correct one for two-mode interference, then the difference in attenuation rates for the two modes is $0.76 \mathrm{~dB} / 1000 \mathrm{~km}$. Experimental values for the attenuation rates of mode 1 and mode 2 for predominantly sea water paths at night are given by Watt and Croghan [1964] for a range of frequencies in the VLF band. At $18.0 \mathrm{kc} / \mathrm{s}$ they give a difference in attenuation rate between mode 1 and mode 2 of 1.5 $\mathrm{dB} / 1000 \mathrm{~km}$. Theoretical values for the difference in attenuation rate between mode 1 and mode 2 will depend on the earth-ionosphere model chosen. Figure 9 shows the difference in attenuation rate between mode 1 and mode 2 at $18.0 \mathrm{kc} / \mathrm{s}$ as a function of reference height $h_{0}$, for a profile of $\omega_{r}$ varying as $2.5 \times 10^{5}$ $\exp 0.5\left(h-h_{0}\right) \mathrm{sec}^{-1}$ and a perfectly conducting spherical earth [Wait and Spies, 1964]. At a reference height of $h_{0}=90 \mathrm{~km}$ the difference in attenuation rates is $1.9 \mathrm{~dB} / 1000 \mathrm{~km}$ when using this particular model. However, the value of $\omega_{r}=2.5 \times 10^{5} \mathrm{sec}^{-1}$ at the reference height $h=h_{0}$, while appropriate for daytime conditions, may not apply at night. A change in this parameter, or of the exponential factor of 0.5 , could lead to differences in attenuation rate in agreement with the experimental value obtained here.

If we again take the slope for distances of greater than $5000 \mathrm{~km}$ as being correct, then the intercept at $d_{N}=0$ gives $20 \log _{10}\left|\Lambda_{1} T_{11}\right| /\left|\Lambda_{2} T_{21}\right|=2.8$. Once again, theoretical values of excitation factor depend on the earth-ionosphere model chosen. Thus for a perfectly conducting spherical earth and a perfectly reflecting ionosphere, height $90 \mathrm{~km}$, Wait [1962a] arrives at a figure of $20 \log _{10}\left|\Lambda_{2} / \Lambda_{1}\right|=12$ at $18.0 \mathrm{kc} / \mathrm{s}$. For a perfectly conducting spherical earth and for a profile of $\omega_{r}$ varying as $2.5 \times 10^{5} \exp 0.5\left(h-h_{0}\right) \mathrm{sec}^{-1}$, and $h_{0}=90 \mathrm{~km}$, Wait and Spies [1964] give $20 \log _{10}$ $\left|\Lambda_{2} / \Lambda_{1}\right|=9.6$ at $18.0 \mathrm{kc} / \mathrm{s}$. These two values of excitation factor, taken together with the experimental value of $20 \log _{10}\left|\Lambda_{1} T_{11} / \Lambda_{2} T_{21}\right|=2.8$, give values of $\left|T_{11} / T_{21}\right|$ of 5.5 and 4.2, respectively. This implies that the conversion from mode 1 in darkness to mode 1 in daylight is approximately five times greater than the conversion from mode 2 in darkness to mode 1 in daylight, at the dawn discontinuity at a frequency of $18.0 \mathrm{kc} / \mathrm{s}$. W ait [1962b] considers the mode conversion at a wedge discontinuity in a parallel plate waveguide with perfectly conducting walls. For the first change of section, i.e., parallel section to wedge section, Wait gives $T_{21} / T_{11} \doteqdot-3 k h \psi / 4 \pi^{2}$, and for the second change of section, i.e., wedge section to parallel section, $T_{21} / T_{11}$ $\doteqdot+3 k(h+\triangle h) \psi / 4 \pi^{2}$, for $k h \psi<<1$, where $k=2 \pi / \lambda$, $h, h+\triangle h$, are heights of two parallel sections, and $\psi=$ wedge angle. Thus the maximum possible conversion for the whole wedge discontinuity, assuming in-phase conditions and ignoring attenuation in the wedge section, would be $\left|T_{21} / T_{11}\right|=3 k(2 h+\triangle h) \psi / 4 \pi^{2}$. For $h=70 \mathrm{~km}, \triangle h=20 \mathrm{~km}$, and $\psi=1 / 50$, this gives $\left|T_{11} / T_{21}\right| \doteqdot 11.4$. Bahar and Wait [1964] also consider the mode conversion at a step discontinuity in a parallel section guide with perfectly conducting walls. This work predicts that the conversion coefficient $T_{2 n}$ for night-to-day transmission should be given by

$$
T_{2 n} \doteqdot \frac{4}{\pi}(-1)^{n} \sin \left(\frac{\pi}{2} \frac{\triangle h}{h}\right) \frac{2 n-1}{(2 n-1)^{2}-\left(\frac{h-\triangle h}{h}\right)^{2}}
$$

where $\triangle h$ is the abrupt height change. For $n=1$, $h=90$, and $\triangle h=20$, we have $\left|T_{21}\right| \doteqdot 1 / 6.4$. Since $T_{11}$ is approximately equal to 1 , this gives $\left|T_{11} / T_{21}\right| \doteqdot 6$.

The single experimental value obtained for $\left|T_{11} / T_{21}\right|$ is therefore of the same order as a theoretical estimate for a step change in ionosphere height in a parallel section guide with perfectly conducting walls, even though the wedge model is a more realistic one.

It is worth noting that if extreme values for the month of December instead of mean values were used in the above calculations, then the resulting difference in attenuation rate for mode 1 and mode 2 would be 1.7 $\mathrm{dB} / 1000 \mathrm{~km}$, and the mode conversion factor ratio would be $20 \log _{10}\left|T_{11} / T_{21}\right|=9.5$ or $\left|T_{11} / T_{21}\right|=2.99$. 
At sunset, similar reasoning gives

$$
\frac{E_{\mathrm{MAX}}+E_{\mathrm{MIN}}}{E_{\mathrm{MAX}}-E_{\mathrm{MIN}}}=\left|\frac{U_{11}}{U_{12}}\right| e^{d_{N}\left(\alpha_{N 2}-\alpha_{N 1}\right)},
$$

where $E_{\mathrm{MAX}}$ and $E_{\mathrm{MIN}}$ are again estimated for a constant distance $d_{N}$ of the dusk line from the receiver. The depths of the minima at sunset were difficult to read on the Nairobi recordings because of the local interference mentioned earlier, and hence the results from the shorter path to H.M.S. Vidal had to be used. Mean values could not be taken because of the movement of the ship, so individual day's recordings were used. The figure obtained for the difference in attenuation rates for the two modes was approximately 0.5 $\mathrm{dB} / 1000 \mathrm{~km}$, i.e., lower than in the dawn case, and the ratio of the amplitudes of the conversion factors, i.e., $\left|U_{11} / U_{12}\right|$, was $2.4: 1$. These results were taken from a few day's recordings and are only approximate.

\section{Conclusions}

The experimental evidence in the paper supports the ionosphere model put forward by Crombie to explain the dawn and dusk phase steps and amplitude fading, i.e., that two waveguide modes exist in the dark part of the path, but only one in daylight. It has been shown that at dawn, distant observers experience the amplitude fading simultaneously, whereas at dusk the time of fading depends on the distance between the receiver and the dusk line. Both these results are for a west-to-east path, effectively normal to the dawn-dusk line (the angle varied between $57^{\circ}$ and $80^{\circ}$ ), and for a frequency of $18.0 \mathrm{kc} / \mathrm{s}$. In addition to this, the difference in phase velocity of the two interfering modes, deduced from the distance moved by the dawn-dusk line between the times of minimum signal, is compatible with the two modes being present under a nighttime height of ionosphere. This again is in agreement with the proposed model.

A technique has been demonstrated for obtaining the difference in attenuation rate for the two principal modes at night. The experimental value obtained at $18.0 \mathrm{kc} / \mathrm{s}$ is lower than that given by Watt and Croghan [1964] for other experimental results. By assuming values for the transmitter excitation factors of mode 1 and mode 2 at night, a figure is given for the ratio of the mode conversion coefficients of mode 1 and mode 2 at the dawn discontinuity. At the dusk discontinuity, the ratio of the mode conversion coefficients is found independently of the transmitter excitation factors. The experimental value at dawn is of the same order as the mode conversion coefficient ratio as predicted by Bahar and Wait [1964] for an abrupt discontinuity in a parallel section guide of infinite conductivity, and is therefore surprisingly high. At dusk the experimental data was not good enough to give a reliable result. The experimental values quoted above are of course for one path, at one frequency, and are based on one month's observations. It is felt that a fuller investigation is needed before deciding on the validity of the technique.
It should also be pointed out that the numerical results outlined above depend on the assumption that the magnitude of the mode conversion is independent of the distance of the dawn-dusk line from the transmitter. If, however, the mode conversion depends on the angle between the path and the dawn-dusk line, then the assumption will not be valid, since this angle changes along the path. Also, Crombie [1964] suggested the possibility that mode conversion does not all take place at the discontinuity, which again would modify the results.

It is proposed to investigate the phenomenon of phase steps and amplitude fading for east-to-west paths, for other frequencies in the VLF band, and to study the effect of angle of path to the dawn-dusk line on mode conversion. In the case of east-to-west paths, the sunset minima should be experienced simultaneously by observers to the west of the discontinuity, whereas at sunrise, the time of minimum signal should depend on the distance between the dawn line and the receiver.

Acknowledgement is made to the Ministry of Defence Hydrographic Department for permission to install the monitoring equipment on board H.M.S. Vidal, and to J. Beaumont of Cambridge University Department of Geodesy and Geophysics for ensuring the high quality of the records obtained. The Nairobi records were taken at the University of East Africa, The Royal College, Nairobi, Kenya.

\section{References}

Anderson, C. N. (1928), Correlation of long-wave transatlantic radio transmission with other factors affected by solar activity, Proc. IRE 16, 297-347.

Bahar, E., and J. R. Wait (1964), Microwave model techniques to study VLF radio propagation in the earth-ionosphere waveguide, Quasi-Optics, ed. J. Fox, 447-464 (Polytechnic Press, Polytechnic Inst. of Brooklyn, N.Y.).

Budden, K. G. (1961), The Waveguide Mode Theory of Wave Propagation (Prentice Hall, Inc., New York, N.Y.).

Crombie, D. D. (1964), Periodic fading of VLF signals received over long paths during sunrise and sunset, Radio Sci. J. Res. NBS 68D, No. 1, 27-35.

H. M. Nautical Almanac Office (1961), The Astronomical Ephemeris for 1963 (Her Majesty's Stationery Office, London).

Pierce, J. A. (1955), The diurnal carrier-phase variation of a 16-kilocycle transatlantic signal, Proc. IRE 43, 584-588.

Rieker, J. (1963), Sunset and sunrise in the ionosphere: effects on the propagation of long waves, J. Res. NBS 66D (Radio Prop.), No. 2, 119-138.

Wait, J. R. (1962a), Electromagnetic Waves in Stratified Media (Pergamon Press, Oxford).

Wait, J. R. (1962b), An analysis of VLF mode propagation for a variable ionosphere height, J. Res. NBS 66D (Radio Prop.), No. 4, 453-461.

Wait, J. R., and K. P. Spies (1964), Characteristics of the earthionosphere waveguide for VLF radio waves, NBS Tech. Note 300.

Watt, A. D., and R. D. Croghan (1964), Comparison of observed VLF attenuation rates and excitation factors with theory, Radio Sci. J. Res. NBS 68D, No. 1, 1-9.

Yokoyama, E., and I, Tanimura (1933), Some long-distance transmission phenomena of low frequency waves, Proc. IRE 21, No. 2, 263-270.

(Paper 69D11-577) 